\title{
VERIFICATION THROUGH THREE-DIMENSIONAL SCANNING OF A PART MADE BY RAPID PROTOTYPING TECHNOLOGIES
}

\author{
Mădălina OPREA ${ }^{1}$, Georgiana Alexandra MOROȘANU ${ }^{1}$, \\ Virgil Gabriel TEODOR ${ }^{1}$, Viorel PAUNOIU ${ }^{1}$ \\ 1 „,Dunărea de Jos University” of Galați, \\ email: Alexandra.costin@ugal.ro
}

\begin{abstract}
Lost wax casting is a known process for making complex shaped parts. Based on the known technology, one can imagine a technological variant that involves obtaining the model through rapid prototyping processes, and the mold will be made around this model. Subsequently, the model, made of a soluble material will be dissolved, leaving the mold with the corresponding inner cavity. A frequently encountered problem is the need to verify the dimensional accuracy of the model, especially in conditions where its complexity is special.

The paper presents the design of the model starting from the shape of the piece to be obtained, the realization of the model using the technology of filament deposition in a molten state and the inspection of the model obtained using a video scanning system.
\end{abstract}

KEYWORDS: rapid prototyping, video scanning, reverse engineering.

\section{INTRODUCTION}

Lost wax casting is a process that uses a wax pattern to create a mold for making a piece or semi-finished product $[1,2]$.

The process has been known over the years as „lost wax casting” or „precision casting” due to its accuracy in creating parts with precise tolerances. The process is used as an alternative for injection molding.

In its industrial form, injection molding is a common way to create precision metal parts.

Designers can combine the lost wax molding process with computer-aided design (CAD) and rapid prototyping technologies to simplify workflow, save time, reduce costs, and modernize the process [3].

\section{DESCRIPTION OF THE CASTING PROCESS}

The lost wax casting process may differ depending on the industry and application, but it generally consists of the following steps [1]:

1. 3D printing of the model: a CAD model containing the geometry of the part is sent to a 3D printer to be made of PVA filament (special plastic that is soluble in water).
2. Assembling the models: the printed models are mounted on the casting channels, being ready for immersion.

3. Slurry coating: the assembly is immersed in a slurry bath to form a shell around it.

4. Sanding: after slurring, sand particles are sprayed on its surface. This helps to thicken and strengthen the coating on the surface of the model.

5. Repeat steps 3 and 4: repeat steps 3 and 4 until the desired shell thickness is reached. The assembly is allowed to set and dry.

6. Disposal of the model: the assembly is immersed in water to dissolve the model.

7. Metal casting: the metal is melted in an oven and then carefully poured into the shell. The coating must be hot because otherwise, the temperature difference would destroy it. The stuffed shells are then allowed to cool.

8. Removing the shell: the material of the shell is removed by hammering, vibrating, or blasting.

9. Cutting: the finished parts are cut from the casting channels; the material will be reused in another casting.

10. Finishing: the castings are machined or sandblasted to obtain the final geometry and surface finish. Where necessary, castings are also heat treated to improve the mechanical properties of the material. 


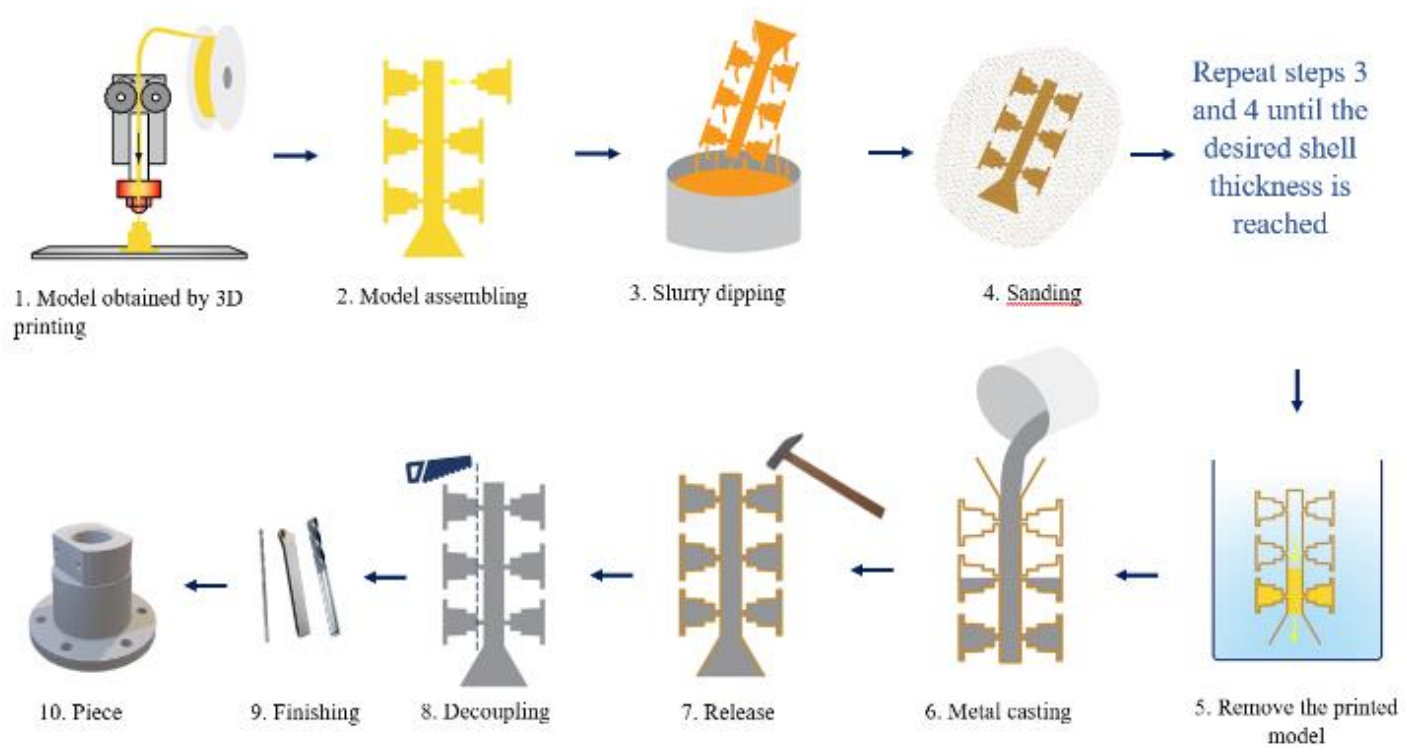

Fig. 1. Lost wax casting process

\section{VERIFICATION OF THE OBTAINED MODEL}

The verification of the model was performed by 3D scanning, using an Athos 500 professional system [4, 5].

3D scanning is the process of analyzing a realworld object to collect data on its shape, structure, and appearance so that it can be used later to build digital 3D models.

With the help of 3D measuring systems, the measurement time in design, manufacture, assembly, and production is substantially reduced.

These systems are based on a 3D optical scanner without physical contact. The purpose of this scanner is usually to create a $3 \mathrm{D}$ model, initially consisting of a "point cloud" obtained from the surface of the scanned object, then used to reconstruct it.

A detailed image, consisting of a large number of measuring points, can be recorded in a few seconds and is calculated by dedicated software, which can then be compared with the theoretical model.

The dimensional accuracy of 3D metering systems is at the micron level, providing fast scanning, even in optically difficult areas (e.g. flutes, bores, etc.) and high-quality data.

Thus, to verify the accuracy with which the "Coupling flange" part was made, the Atos Core device, figure 2, and GOM equipment using modern and innovative technology for the fully automated inspection and measurement of the part were used.

Measurement systems are based on digital image processing and are used in product development and quality assurance, testing of materials and components $[6,7]$.

The Atos series of contactless industrial 3D scanners, with narrowband blue light, offer precision scans with detailed resolution at high speeds. Atos is designed with advanced hardware and intelligent software for accurate, repeatable measurements with flexibility and process reliability [4].

Atos solutions are used in many industries for parts testing, such as sheet metal parts, tools and dies, turbines, prototypes, and injection molded and diecast parts.

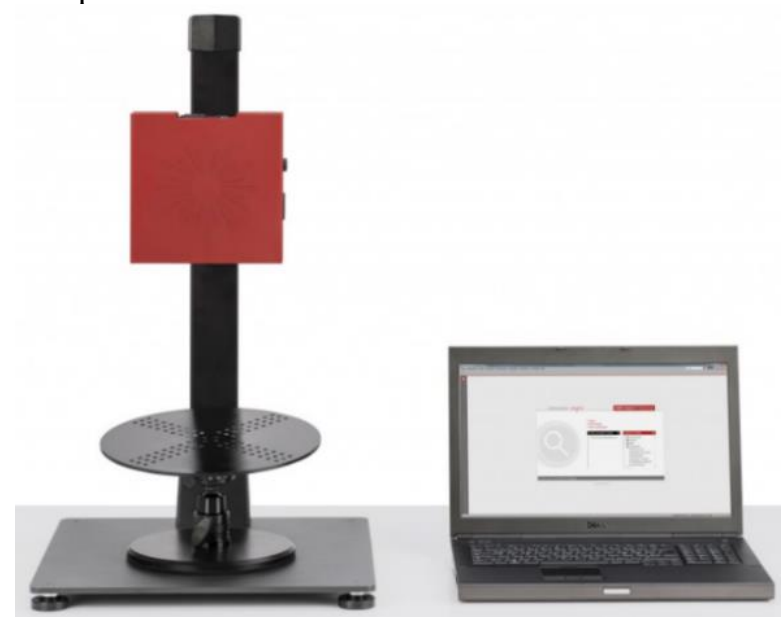

Fig. 2. Atos Core 500 Scanner [5]

Atos Core specializes in the three-dimensional measurement of components up to $500 \mathrm{~mm}$ in size. The sensor forms the basis for a wide range of measurement tasks - from simple 3D scanning to fully automated measurement and inspection processes.

The projection unit in the Atos scanner is based on "Blue Light" technology, which means that the scanner projects light from a narrowband blue frequency. Thus, the ambient light is filtered during the scanning process and does not interfere during the measurement. The strong blue light source results in an efficient and fast measurement [5]. 


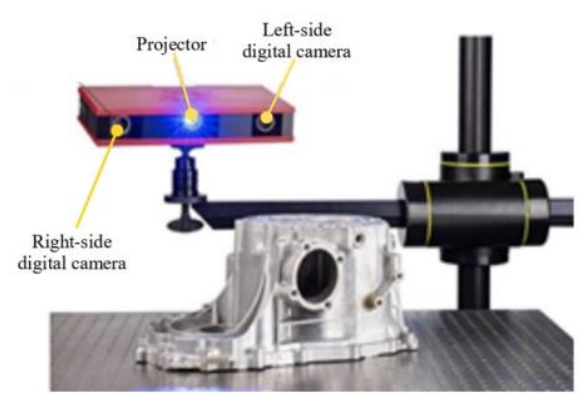

Fig. 3. Composition of the Atos Core 500 scanner [5]

The Atos scanner has three main components in its structure, figure 3: two cameras - left and right and a projector.

Atos Core uses a set of stereo cameras that work on the principle of triangulation, figure 4 .

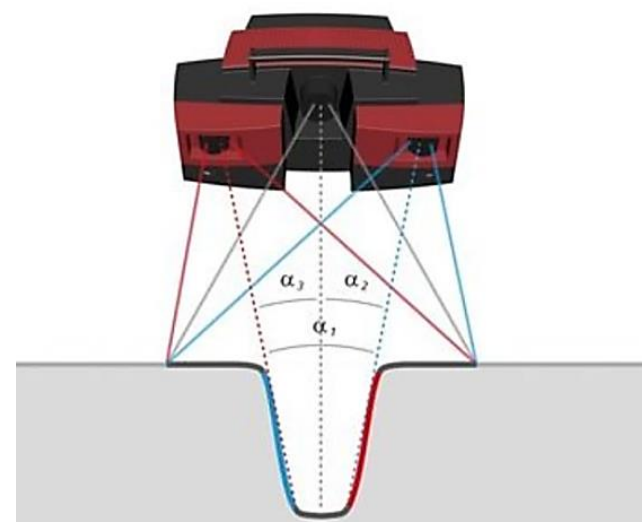

Fig. 4. Principle of triangulation [5]
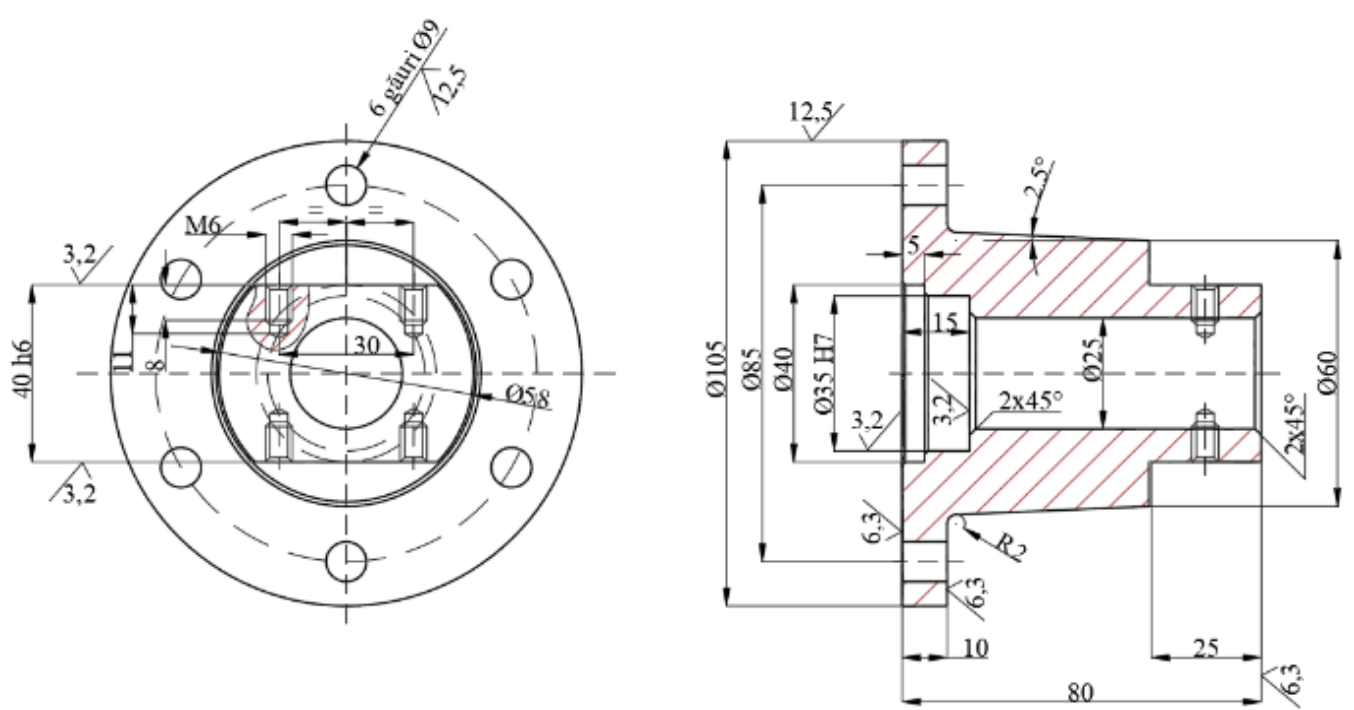

Fig. 6. Execution drawing of the "Coupling flange" part

For the Atos Core to cover the entire surface of the part, the scan must be done from several positions.

To start the scanning process, it is necessary to apply reference markers, both on the table of the scanning equipment, figure $7 . a$, and on the part, figure
The term "triple scanning technology" refers to the fact that three different methods are used to capture data during the scanning process [5]:

1. Camera left / Camera right;

2. Left Camera / Projector;

3. Right Camera / Projector.

Because both the camera and the projector are calibrated, all three elements are used to calculate the coordinates.

\section{3D SCANNING OF THE "COUPLING FLANGE"}

In order to verify the accuracy of the model, the part made of PLA will be scanned, using the Athos Core 500 system, figure 5 .

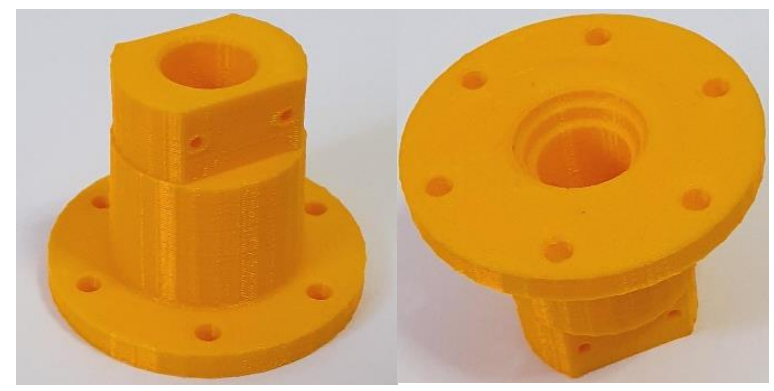

Fig. 5. Piesa „Flanșă de cuplare”

Figure 6 shows the execution drawing of the "Coupling flange" part. 7.b, so that they can be recognized by the scanner as reference points.

For a more accurate scan of the surfaces of the parts and in order that the device can capture as many details as possible, from all angles, after each scan, 
the table on which the part is positioned will be revolved until it makes a complete rotation.

Thus, for the top view of the piece "Coupling flange" 25 scans resulted, and for the bottom view, 10 scans, figure 8 .

After the two scanned positions, the polygonization process begins. This consists in correlating all the scans of the part and obtaining the complete numerical model.

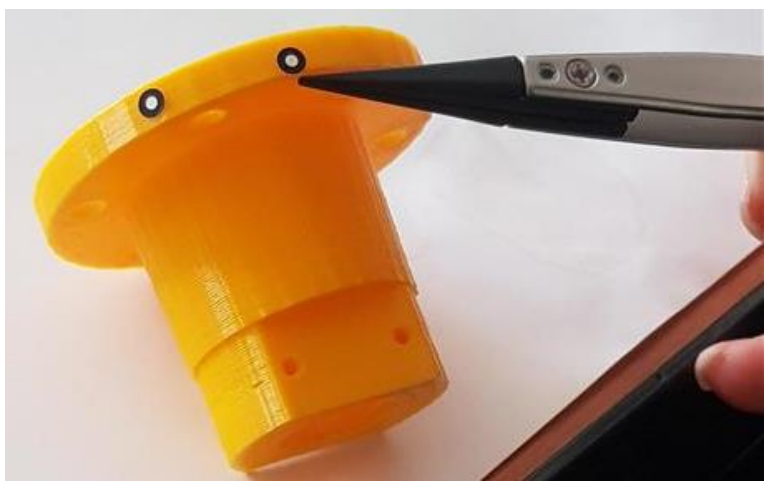

a).

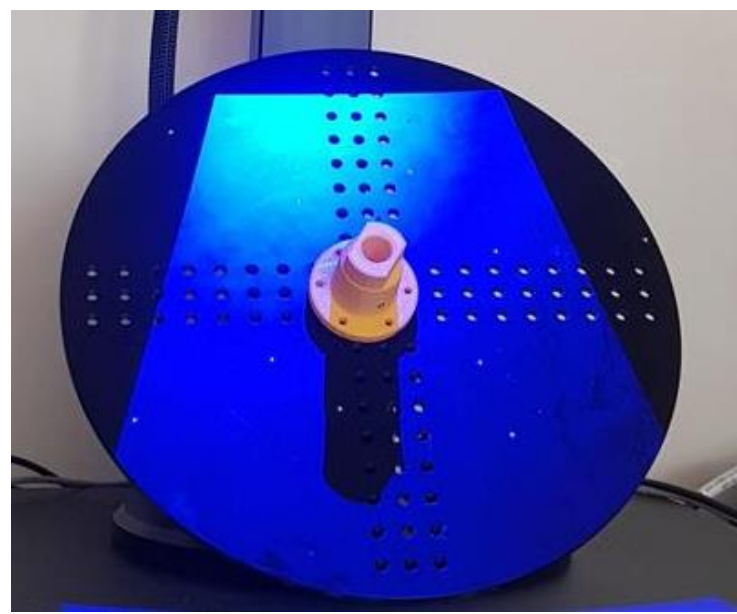

b).

Fig. 7. Application of reference markers: $a-$ on piece; $b$ - on the equipment table

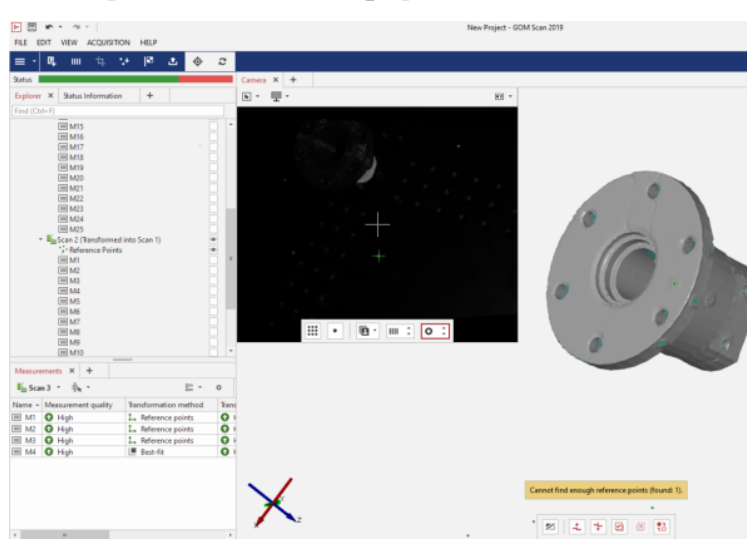

Fig. 8. Number of scans for each position of the "Coupling flange" part
Thus, from the GOM menu choose: Aquisition $\rightarrow$ Measure series $\rightarrow$ Polygonize and Recalculate and calculate the network of points provided on the surface of the part. Imperfections and unwanted edges on the scanned part will be removed with the Select / Deselect Through Surface command. Figure 9 shows the final model of the "Coupling flange" part, scanned using the Atos Core equipment.

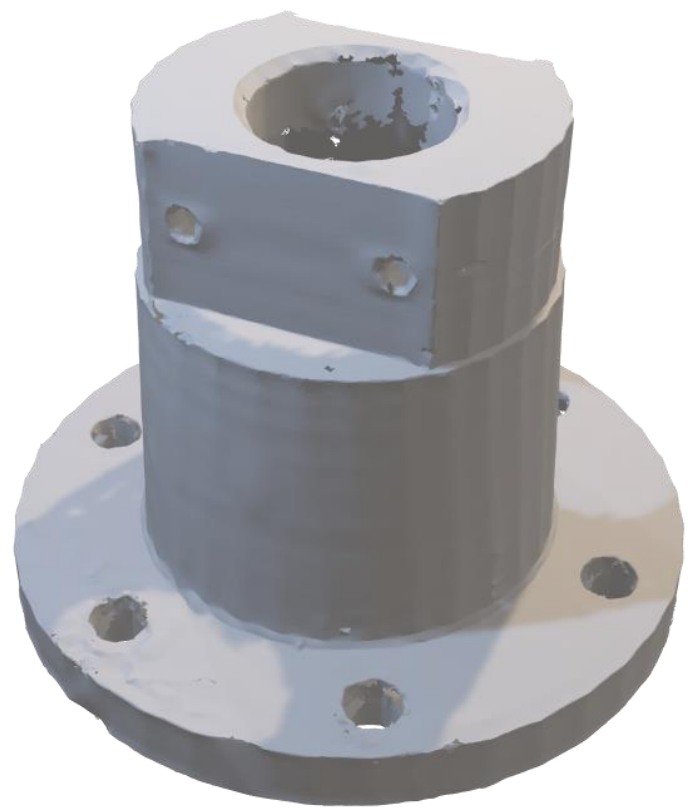

Fig. 8. Scanned model of the "Coupling flange" part

The scanned part will be saved in *.stl format, thus allowing its opening in various software design programs, such as Autodesk Inventor, AutoCAD, Catia, SolidWorks, etc.

To verify the efficiency of the Atos Core scanner, it is necessary to model the "Coupling flange" in a 3D graphic design program, followed by the comparison between the two models obtained - the scanned model and the CAD model, and establishing the accuracy of the model.

Thus, it is chosen that the "Coupling Flange" part is modeled in the Autodesk AutoCAD program, a software package that makes the $3 \mathrm{D}$ modeling of solids as easy and precise as possible.

The CAD model of the "Coupling Flange" part made using the Autodesk AutoCAD software is shown in Figure 9. 


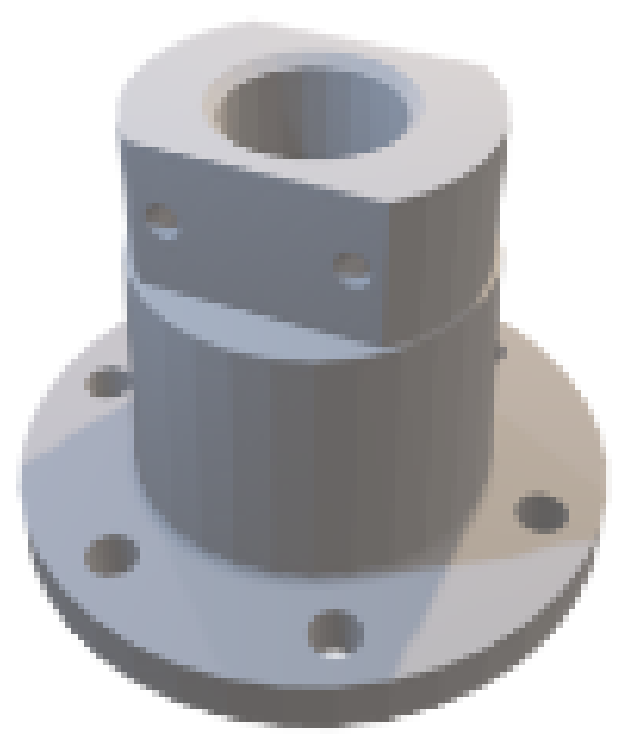

Fig. 9. CAD model of the "Coupling flange" part

The model thus obtained will be saved in *.stp format, to allow its inspection in the GOM Inspect program.

\section{INSPECTION OF THE SCANNED MODEL AND COMPARISON WITH THE CAD MODEL OF THE PART}

The GOM Inspect application opens and the two models of the "Coupling flange" part are imported from the File $\rightarrow$ New Project menu - the scanned model, in *.stl format and the CAD model, in * .stp format. Initially, the two models are displayed with different orientations in relation to the reference system prescribed in the program, figure 10.

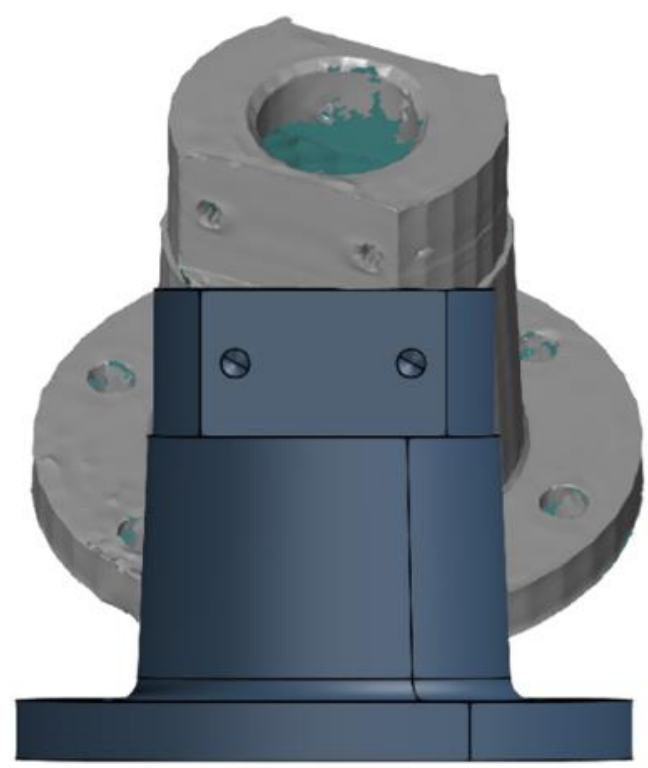

Fig. 10. Import the scanned model and CAD model into the GOM Inspect program
To overlap the two models in a single reference system, access the menu Operations $\rightarrow$ Alignment $\rightarrow$ Initial Alignment $\rightarrow$ Prealignment and obtain the model shown in Figure 11.

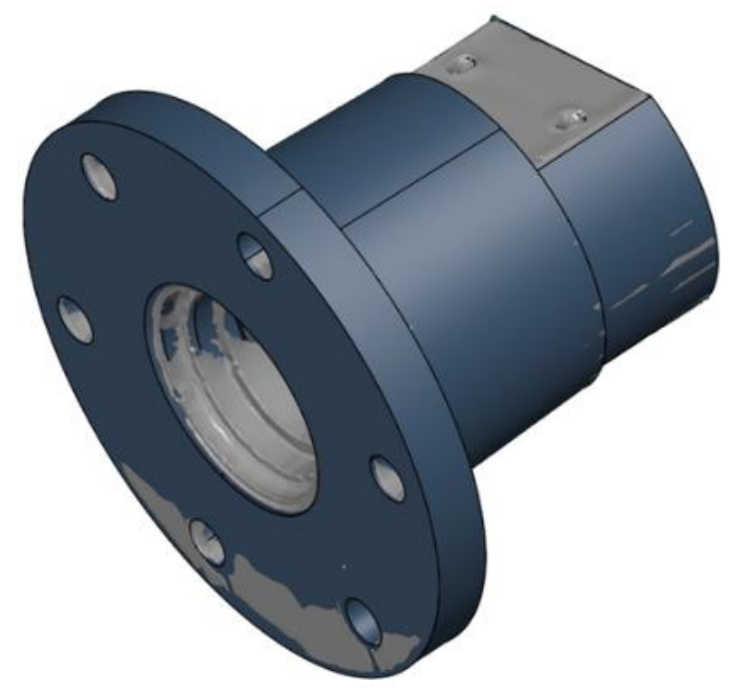

Fig. 11. Overlap of the two models of the "Coupling flange" part

The inspection of the two models begins and, in this case, it is necessary to access the menu Inspection $\rightarrow$ CAD Comparison $\rightarrow$ Surface Comparison on $C A D$. The inspection, which analyzes the imperfections of the "Coupling flange" part, compared to the numerical model, is done by comparing the surfaces and representing the deviations, using a colors code, figure 12.

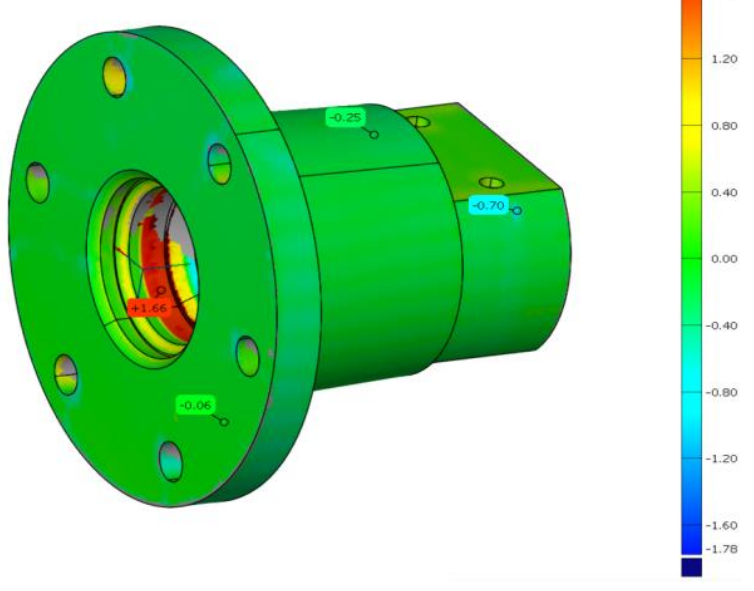

Fig. 12. The result of the inspection on the $C A D$ model

The color code used to represent the deviations is as follows:

- green $\rightarrow$ deviations of $\pm 0,40 \mathrm{~mm}$;

- cyan $\rightarrow$ deviations of -0,4 și - 0,8 mm;

- blue $\rightarrow$ deviations of ntre $-0,8$ și $-1,78 \mathrm{~mm}$;

- yellow $\rightarrow$ deviations of $+0,4$ și $+0,80 \mathrm{~mm}$.

- red $\rightarrow$ deviations of $+0,8$ și $+1,86 \mathrm{~mm}$; 


\section{CONCLUSIONS}

Inspection of part deviations indicates the following:

- small deviations, of $\pm 0,40 \mathrm{~mm}$, for $90 \%$ of piece's surface;

- lower deviations, of medium level (between -0.4 and $-0.8 \mathrm{~mm}$ ), which appear in the areas where the inspection indicates lack of material;

- lower deviations, high level (between -0.8 and $1.78 \mathrm{~mm}$ ), which occur in areas where the inspection was difficult to perform;

- upper deviations, of medium level (between +0.4 and $+0.8 \mathrm{~mm}$ ), which appear in the areas where the inspection indicates surplus material;

- superior, high-level deviations (between +0.8 and $+1.86 \mathrm{~mm}$ ), which occur in areas with an accentuated surplus of material.

After inspecting the "Coupling Flange" part, using the comparison on the surface of the CAD model, it was found that the model made by rapid prototyping is sufficiently accurate, and the inspection using the Athos Core 500 system completely covers the surface of the scanned part and offers very high accuracy all the details of the measured part.

\section{ACKNOWLEDGEMENTS}

This work was supported by the Romanian Ministry of Research and Innovation, CCCDI -UEFISCDI, project number PD-III-P1-1.2-PCCDI-2017-0446 / Intelligent manufacturing technologies for advanced production of parts from automobiles and aeronautics industries (TFI PMAIAA) - 82 PCCDI/2018, within PNCDI III.

\section{REFERENCES}

[1] https://en.wikipedia.org/wiki/Lost-wax casting\#Process

[2] http://americancastingco.com/investment-casting-process/

[3] https://en.wikipedia.org/wiki/3D scanning\#Applications;

[4] http://spectromas.ro/gama-atos-sisteme-de-scanare-3d-noncontact/;

[5] https://www.zebicon.com/en/metrology-systems/3dscanning/atos-core/;

[6] Raja, V., Fernandes, K. J., Reverse Engineering. An Industrial Perspective, Springer-Verlag London Limited, ISBN 978-1-84628-856-2, 2008;

[7] Boboulos, M., CAD-CAM Rapid-prototyping Application Evaluation, Ventus Publishing ApS, ISBN 978-87-7681-676-6, 2010; 